\title{
Plantar puncture wounds: a survey to determine the incidence of infection
}

Division of Emergency Medicine, University of California, San Francisco, USA E J Weber

Correspondence to: Ellen J Weber MD, Box 0208 L138, Emergency Department, University of California San Francisco, San Francisco, CA 94143-0208, USA

Accepted for publication 16 November 1995

\begin{abstract}
Objective-To determine the incidence of plantar puncture wound infections. Methods-Ambulatory emergency patients were surveyed with regard to a previous history of plantar puncture wounds and any subsequent infections.

Results-200 questionnaires were analysed. Forty four percent of respondents had previously sustained at least one plantar puncture wound. Of 156 wounds, $79(50 \%)$ came to the attention of a physician. There were 10 infections, nine of which were seen by a physician. Counting only those wounds seen by a physician, the apparent infection rate in the study population is $11.4 \%$. When all wounds are included, the infection rate is $6.4 \%$.

Conclusions-The infection rate of plantar puncture wounds is lower than most studies report because many wounds with a benign outcome never come to medical attention. Recommendations for treatment of fresh puncture wounds in the emergency department should be evaluated in light of this infection rate.

( $\mathcal{F}$ Accid Emerg Med 1996;13:274-277)
\end{abstract}

Key terms: foot wounds; osteomyelitis; punctures; wound infection

Plantar puncture wounds are common injuries, but the appropriate treatment for fresh wounds has not been established. Recommendations for treatment to prevent subsequent infection range from simple cleansing and tetanus booster $^{1}$ to various combinations of $x$ rays, debridement, probing, surgical exploration, and drains. ${ }^{2-7}$ Elaborate scoring systems have been devised to help the emergency physician determine just how aggressive to be. ${ }^{8}$

Recommendations for aggressive wound care are based on the concern that plantar puncture wounds have a high rate of infection and complication. However, there are few published data to support this view. Three previous series $^{349}$ have suggested an infection rate of $10-12 \%$. Most other reports of puncture wound complications are series of cases referred for treatment. It has been suggested that many people who incur these wounds never seek treatment, ${ }^{8}$ so that benign outcomes are not included in calculation of infection rates.

If the infection rate of plantar puncture wounds is, in reality, not very high, the aggressive emergency treatment of fresh wounds that is often recommended may not be necessary or cost-effective. This study was therefore conducted to determine the frequency of this injury, the percentage of victims who visit a physician for the problem, and the incidence of infection. It was hypothesised that the true infection rate of plantar puncture wounds is smaller than that in published series because so many uninfected wounds are never reported.

\section{Methods}

The study was conducted in the emergency department at the University of California, San Francisco, during the two month period between 15 March and 15 May 1992. A simple survey written in English, consisting of six multiple choice questions and a request for the respondent's age, was distributed to all study patients (fig 1). All ambulatory patients who presented to the emergency department were eligible for inclusion in the study. Surveys were distributed by the triage nurse at the time of triage; patients returned the survey to the triage nurse before being called into the department for the examination. Patients whom the nurse felt were too ill to complete a survey were excluded. On certain shifts, the survey was not distributed at all because the department was too busy or because the triage nurse was not a regular staff member and had not been instructed to distribute the survey. NonEnglish speaking patients were also excluded. Children who were registered patients and could read and write were allowed to complete the survey with aid of a parent; otherwise, a parent did it for them.

\section{Results}

All patients who were asked to complete the survey did so, for a total of 201 responses. One survey was excluded from analysis because of bizarre responses (psychiatric patient). Of the remaining 200,88 had incurred one or more plantar puncture wounds (44\%) and 112 had not $(56 \%)$. Respondents' ages ranged from 4 to 81 years; the median age was 35 .

One hundred and fifty six wounds were reported. Eight wounds had become infected. In one additional survey, two wounds and two physician visits were reported in questions 2 and 3. The respondent answered "yes" to the question of whether any of the wounds had become infected, but did not state how many had become infected or whether they had seen a physician for the infection(s). It was felt that this survey should be included in the study with the assumption that the respondent had two wounds with two infections, for a total of 10 infections in the sample overall.

One hundred and forty five wounds did not become infected. Included in this category were the surveys of seven respondents (nine 
Dear Patient,

We are conducting an informal survey to determine some facts about a common medical problem. Would you please take a moment to answer the questions below? Then return the survey to the nurse who gave it to you. All answers are confidential. Thank you.

1. Have you ever stepped on a nail that entered the bottom of your foot?

$$
\text { Yes No }
$$

2. How many times would you say this has happened to you?

0

1

2

3 or more times

3. How many times would you say you saw a doctor for this kind of injury?

0

1

2

3 or more times

4. Did you ever get an infection from this kind of injury?

Yes No

5. If yes, how many times did you have an infection?

0

1

2

3 or more times

6. Did you have to see a doctor for any of these infections? If so, how many times?

I never had to see a doctor.

I saw a doctor for one infection.

I saw a doctor for two infections.

I saw a doctor for three or more infections.

7. How old are you?

Thank you.

Figure 1 Puncture wound survey.

wounds) whose responses were not entirely consistent. These respondents answered "No" to the question of whether any of their wounds had become infected and did not circle any answer in question 5 (indicating no wounds became infected), yet each circled the response that they had seen a physician for a single infection in question 6 . In calculating the infection rate, our assumption was that these respondents misunderstood question 6 as either referring to any wound infection or to the visit for the wound itself. As a result, these wounds were considered to be uninfected.

The number of wounds that were seen by physicians was calculated from the response to question 3 . Fifty percent (79/156) of wounds were reported to physicians (question 3); nine of 10 infections were seen by physicians. No patient required more than one visit to a physician for an infection. Looking only at the wounds that were reported to physicians, the infection rate of plantar puncture wounds appears to be $11 \cdot 4 \%$. However, when the wounds that never came to the attention of a physician are also included, there were 10 infections in 156 wounds, giving an infection rate of $6.4 \%$ (fig 2 ).

\section{Discussion}

When faced with fresh traumatic wounds, emergency physicians must weigh a number of factors in deciding how aggressive to be in their treatment. How high is the risk of infection? How invasive or risky is the treatment? What 
Incidence of puncture wound infections

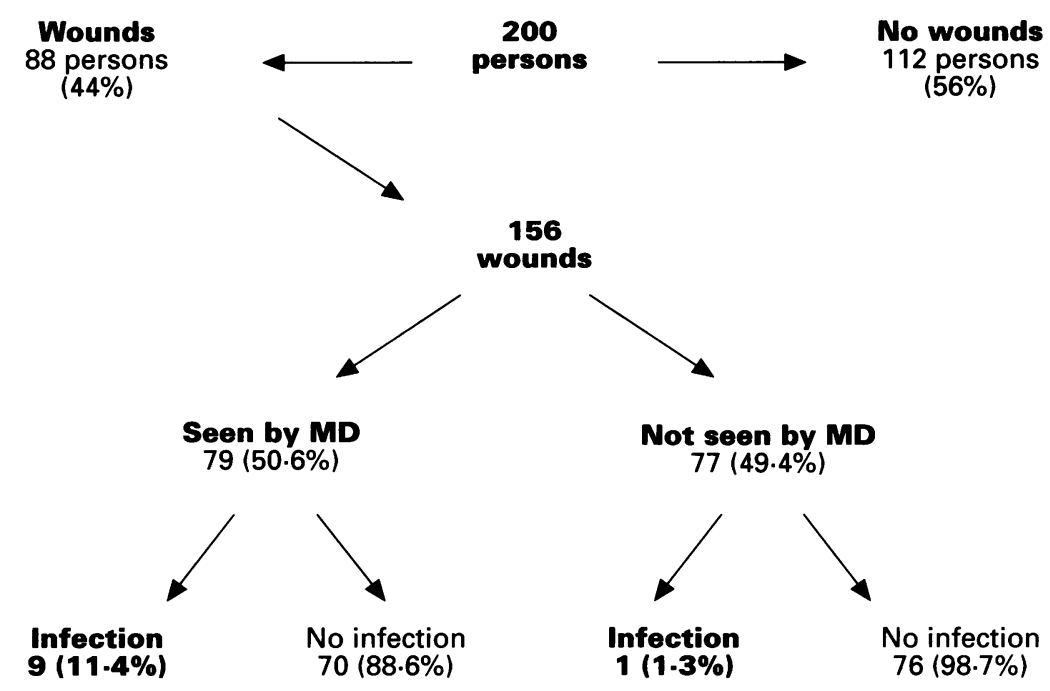

Actual incidence of infection $6.4 \%$ (10/156)

Figure 2 Incidence of puncture wound infections in the study population. The incidence is almost twice as high if only those subjects who saw physicians are included in the calculations. wounds and ultimately developed an infection. Since patients with infections are more likely to come to the emergency department than those who experience no problems with this wound, this infection rate is likely to be falsely increased. Indeed, in an earlier study, Houston reviewed 2583 puncture wounds that presented to his emergency department and found that $10 \%$ of patients who presented late had infections. However, among those who presented to the emergency department without infections, only $2 \%$ subsequently became infected. ${ }^{4}$

Sixteen patients in Fitzgerald's series developed osteomyelitis; the reported osteomyelitis rate was $1 \cdot 6 \% .^{3}$ However, only five of these wounds had initially been evaluated in the emergency department. The other 11 had been referred from outside physicians for this complication. The rate of osteomyelitis in the emergency department population was $0.6 \%$. Only two patients in Houston's series developed osteomyelitis, giving a rate of $0.04 \%{ }^{4}$

Unfortunately, neither of these studies looked at the natural history of plantar puncture wounds. Both applied an aggressive surgical wound care protocol to all wounds and neither controlled for the use of antibiotics. Thus it is not known what the real infection rate would have been without such aggressive treatment, or whether the treatment lowered the infection rate.

More recently, Schwab and Powers prospectively studied 63 emergency department patients with plantar puncture wounds who were treated conservatively. ${ }^{9}$ They found an infection rate of $8 \%$ and a complication rate of $12 \%$.

My present study hypothesised that the infection rate of plantar puncture wounds has been overestimated in studies that combine infected patients with newly injured patients, or look only at patients who present for treatment, ignoring those who never see a physician because they do not develop complications. ${ }^{8}$

My study has shown that plantar puncture wounds are a very common injury (nearly $50 \%$ of the sample had incurred this injury) and that half of the victims never seek medical attention. In the study population, the overall infection rate for all victims of this injury was $6 \cdot 4 \%$, a figure comparable to that of clean wounds sutured in the emergency department. ${ }^{10}{ }^{11}$ No patient reported more than one physician visit per wound infection, indicating a low complication rate. The infection rate appears much higher if one looks at only those wounds that were seen by a physician, suggesting that conclusions regarding infection rates based on doctor's visits may not always be accurate. Given this relatively low infection rate, emergency physicians must begin to re-examine the need for invasive, painful, costly and time consuming wound care for acute plantar puncture wounds.

There are several limitations to this type of study. For one thing, it is a retrospective survey which runs the risk of memory bias for the respondents. However, patients are more likely to forget a puncture wound with a benign senting to the emergency department within 24 hours. However, this infection rate included patients who presented with established infections as well as those who presented with fresh 
course and remember it if there was a complication. Thus, if anything, the survey method would tend to overestimate the incidence of infection.

Another concern is that patients are not consistently capable of diagnosing their own wound infections, ${ }^{11}$ so that the infection rate may be underestimated in this study. By asking patients to recall the number of times they saw a physician as well as the number of infections, it was hoped that most of these infections would be captured even if the patient did not recognise them. It is likely that if patients have a clinically significant infection, particularly one that results in complications (such as osteomyelitis), they would eventually come under a doctor's care.

This study group may not be representative of the general population. All of the respondents were people waiting to be evaluated for another problem at the emergency department. These respondents are individuals who might be more willing to seek care for a medical problem than others. This might mean that our respondents were more likely to have seen physicians for a puncture wound than the general population, and yet only half of them did. On the other hand, they may also be individuals who do not have their own physicians, or ready access to medical care, and so might have been less likely to seek care for these wounds. Nevertheless, that $50 \%$ of the victims never sought medical attention for this injury is entirely consistent with prior estimates. $^{8}$

The survey generated inconsistent responses from eight patients. In one case, it was felt prudent to maximise the number of infections for the respondent who stated he had incurred two wounds and answered in the affirmative to the question of whether they had become infected, but failed to designate the number of infections. For seven patients, it was felt the responses to questions 3-5 clearly indicated they had not had infections from their wounds, even though they had chosen a positive response in question 6 . If these wounds had been included as infected, the overall infection rate in the study would be $19 / 156$ or $12 \%$. This rate still compares favourably with the 5\% infection rate of lacerations sutured in the emergency department. ${ }^{1011}$ Nevertheless, it seems unlikely that these wounds were actually infected, and including them in the calculations as infected would undoubtedly overestimate the infection rate.

In an attempt to keep the survey brief and uncomplicated, there were several areas that were not explored. Did those patients who had infections see a physician before the infection, or only when it became infected? What type of infections did these people have? How were they treated? What percentage had osteomyelitis? Given the potential length of time since the injury, it also did not seem reasonable to ask respondents to recall the depth of the wound or location of the wound on the foot, whether the nail was clean or dirty, or what type of shoes they were wearing, factors which may contribute to the overall risk of infection. ${ }^{6} 812$ Finally, the study did not ascertain the type of initial wound care these patients received, which may have contributed to the incidence of infection we found in this survey.

\section{CONCLUSION}

Plantar puncture wounds are a common injury and many people never see a physician for the problem. The risk of infection is probably no greater than for other types of traumatic wounds seen in the emergency department. Recommendations for invasive wound management for fresh puncture wounds should be considered in light of this risk of infection.

1 Fisher MC, Goldsmith JF, Gilligan PH. Sneakers as a source of Pseudomonas aeruginosa in children with osteomyelitis following puncture wounds. $f$ Pediatr 1985; 106:607-9.

2 Chisholm CD, Schlesser JF. Plantar puncture wounds: controversies and treatment recommendations. Ann Emerg Med 1989;18:1352-7.

3 Fitzgerald R, Cowan J. Puncture wounds of the foot. Orthop Clin North Am 1975;6:965-72.

4 Houston A, Roy W, Faust R. Tetanus prophylaxis in the treatment of puncture wounds of patients in the deep South. F Trauma 1962;2:439-46.

5 Joseph W, LeFrock J. Infections complicating puncture wounds of the foot. F Foot Surg 1987;26:S30-3.

6 Peterson $\mathrm{H}$, Tressler $\mathrm{H}$, Lang A, Johnson E. Osteomyelitis after puncture wounds of the foot. Minn Med 1973; 56:787.

7 Resnick CD, Fallat LM. Puncture wounds: therapeutic considerations and a new classification. $\mathcal{F}$ Foot Surg 1990; 29:147-53.

8 Krych SM, Lavery LA. Puncture wounds and foreign body reactions. Clin Podiatr Med Surg 1990;7:725-31.

9 Schwab R, Powers $R$. Conservative therapy of plantar puncture wounds. $f$ Emerg Med 1995;13:291-5.

10 Galvin J, DeSimone D. Infection rate of simple suturing. $f$ Am Coll Emerg Physicians 1976;5:332-3.

11 Seaman M, Lammers R. Inability of patients to selfdiagnose wound infections. $\mathcal{F}$ Emerg Med 1991;9:215-9.

12 Patzakis M, Wilkins J, Brien W, Carter V. Wound site as a predictor of complications following deep nail punctures to the foot. West $\mathcal{f}$ Med 1989;150:545-7. 